To appear in: The Cambridge History of Philosophy of the Scientific Revolution, eds. D.M. Miller and D. Jalobeanu.

\title{
From metaphysical principles to dynamical laws
}

\author{
Marius Stan
}

Modern physics depends on dynamical laws; they are the chief components of their respective theories, whose power to explain or predict ultimately comes from them. Generally speaking, dynamical laws are functional dependencies between kinematic and kinetic variables; they are differential equations, relating operators and functions common to a broad variety of motion patterns; and they entail equations of motion-local variants of the laws, adapted to the specifics of some family of systems; e.g., oscillators or elastic gases. These features are strikingly apparent not just in quantum theory, but also in the modern mechanics of classical processes. Dynamical laws are known as the 'cardinal equations' in mechanics that takes force as primitive; and as the 'central equation' in analytic mechanics. ${ }^{\mathrm{I}}$ These laws are then customized so as to yield system-specific, particular equations of motion: e.g., the dynamical equations of a rigid body moving around a fixed point; the Navier-Stokes equation for a viscous fluid; and the Euler-Lagrange equation for a damped oscillator.

This fact-namely, that dynamical laws are differential equations whose main role is to entail equations of motion-was not always the case. From its Cartesian birth in the I640s, classical mechanics was built up from "laws of nature," "laws of motion," or eponymous principles. These early modern laws differed from ours. First, their function was to supply mechanics with a basic ontology, or class of unit bodies. The ontology was articulated prior to the laws - usually, as an extension of substance metaphysics-and then the laws were predicated of this ontology of body. Second, these laws served to channel truth into mechanics - from metaphysics, via the doctrine of body; and from select phenomena of motion, construed as evidence for the laws. However,

\footnotetext{
${ }^{\mathrm{I}}$ Cf. Gallavotti I 983 and Papastavridis 2002.
} 
around $\mathrm{I} 75^{\circ}$ the laws of motion begin to lose both functions. They turned into mere assertions of equality between magnitudes, and served just to entail (differential) equations of motion for specific mechanical situations. Consequently, in the Enlightenment, the laws no longer made claims about natures, and they became epistemically opaque, in that it was no longer clear what counted as strong evidence for the basic laws of mechanics.

This change is among the critical inflection points where early modern mechanics turned into classical mechanics as we know it. This chapter describes and explains the shift, beginning with an account of how the laws of nature linked mechanics to philosophy in the I6oos. Next, I point out an incurable shortcoming in those laws with regard to their role in quantitative theory. Then I explain how the I 70os fixed that shortcoming, and how the solutions thereby changed the logical structure of mechanics. I complete my explanation with some key mathematical notions-born in the I70os, as well-that enabled the changed conception of laws of motion. Briefly put, my thesis is that:

I. the modern concept of laws of motion-qua dynamical laws-emerged in 18 th-century mechanics;

2. the driving factor of this reconceptualization was the need to extend mechanics beyond the centroid theories of the late-I60os; and

3. the development of differential equations enabled the shift.

In addition, I will note how the historical change raised two philosophical difficulties, which ipso facto are extant in the modern concept of dynamical laws: It is unclear what counted as evidence for the laws of motion in the Enlightenment; and it is a mystery whether these laws retain any notion of causality. The latter threatens to subvert the early modern dictum that physics is a science of causes.

Throughout the chapter, I am motivated by two aims. One is to strengthen the case that a "quiet" revolution unfolded through the I70os in then-fundamental science. The other is to stimulate research on the foundations of Enlightenment mechanics. We have excellent studies of natural philosophy in the I60os, but little has been done to determine which central themes and research agendas survived into the following century. Conversely, we have some good studies of I8th-century mechanics, but not much work on how Enlightenment science related to the philosophy that came before it. I expect my paper to serve 
as a specimen of work we could do to bridge the gap between these early-modern areas. $^{2}$

\section{Basic laws in the 1600 s}

My case rests on the premise that key I 7 th-century figures believed that ontology and mechanics must be yoked to each other, via the basic laws of motion. The evidence for this is in plain sight; it just needs coherent assembling.

In Principles of Philosophy, Descartes asserts three "laws of nature" after a summary of his metaphysics and epistemology. ${ }^{3}$ Of these laws, two jointly amount to a conservation principle (of size, shape, and motion-state) for a single body absent an interaction. The third codifies the result of any two-body interactions by contact: it is always the outcome uniquely compatible with the "least modal change" among the involved bodies. ${ }^{4}$ Famously, from these laws he sought (unsuccessfully) to derive the kinematic rules of 2-body impact. But the "modes" in question-really, the three effective parameters privileged by Descartes's first two laws - are just the co-essential properties of all extended substances, or bodies. Therefore, his "laws of nature" straddle the ontology of substance and the basic physics of micro-scale processes. They have epistemological import, too. His evidence for his laws was not empirical. Rather, he rested their truth on alleged facts about the action of God qua creator, its simplicity, and that simplicity's conceptual connection to the modes of extended substance.

The failure of "Descartes and others" to derive sound collision rules drove Leibniz to complain that the error was in their defective laws of nature-and

\footnotetext{
${ }^{2}$ The suggestion that a "quiet" revolution affected mechanics in the I 700 s comes from Hepburn 2013, and Caparrini \& Fraser 2013. Important studies of Enlightenment dynamics are Fraser I983, I985; the technical surveys in Truesdell I960, and his older polemic in Truesdell I968. Calinger 20I6 and Verdun 20I 4 have set the new standard for work on Euler's science.

${ }^{3}$ The account is in Book I of Principles. There were many criteria for substancehood in the I60os-unity, activity, per se conceivability, persistence through change, etc. Descartes' chosen criterion is ontological independence (of anything else). So he infers, correctly, that only God counts as a genuine substance in this sense, and so his metaphysics includes indispensably a philosophical theology. Then he introduces an honorific sense, as it were, of substance qua that which needs no other help but God's to exist. Res extensae thus count as honorific substances; cf. Meditation Two.

${ }^{4}$ The phrase comes from Garber I 992, who explains lucidly the true tenor and content of Descartes's third law. Recent discussions of Descartes' laws, and the foundational debates they caused after him, especially in Britain, are Jalobeanu 20I I and Brading $201 \mathrm{I}$.
} 
ultimately in their metaphysics. So he sought to reform the latter by endowing bodies with forces, or powers "inherent in all corporeal substance as such." His new ontology yielded "systematic laws of motion," which he placed at the basis of a science of motion that he called 'dynamics.' Among these laws was that to every action there is a reaction; and that vis viva (his name for a species of active force) is conserved in all motion exchanges.

Leibniz's epistemology of laws does not yet seem to have been elucidated fully. Still, it is safe to say, the proof-structure of his famous conservation principle is by reductio: unless vis viva is conserved, perpetual motion would ensue, which is impossible. As evidence for his law of action-reaction, he used an informal proof by Jakob Hermann, based on the Principle of Sufficient Reason. ${ }^{5}$ At any rate, Leibniz did not think the fundamental laws are learned from empirical sources; his German successors followed him in this commitment. Their disagreements aside, Leibniz thus concurred with Descartes and Malebranche that the basic laws of motion fall out of a priori metaphysical knowledge.

Few might expect Newton to have endorsed this heavily philosophical, foundationalist picture of laws. And yet, endorse he did-at least in metaphysics, inverting the rationalists' reasoning above. The young Newton had an inchoate metaphysics of body, sketched as a conjectural scenario. In it, God singles out finite volumes of absolute space, and endows them with impenetrability, causal agency on senses like ours, and mobility compatible with the laws of motion. That is, to be a body requires inter alia the power to move as the laws dictate. In effect, Newton at times endorsed a "law-constitutive" account of body. ${ }^{6}$ The laws of motion constrain metaphysical theorizing about corporeal substance-and so they are an explanatory bridge from dynamics to ontology, not the other way around, as his rationalist peers had it. Still, that Newton expects a bridge between them at all shows him quietly subscribing to the 17 th-century picture of the two disciplines yoked together by the fundamental laws of motion.

\footnotetext{
${ }^{5}$ See Leibniz, Specimen dynamicum, WF I63, and also his unpublished piece "On Body and Force, against the Cartesians," in GP IV, 395. His impossibility argument for conservation of vis viva is in the posthumous Dynamica (GM VI, 28I-5I4). For Hermann's law of action and reaction, and Leibniz's endorsement, see Stan 2017.

${ }^{6}$ Brading 20 I I makes a solid case for this reading, and argues that Newton remained committed to a law-constitutive account of body through his late career, well past De gravitatione (where he advanced his conjectural scenario), which is now thought to be a youthful piece; cf. Biener 2017.
} 
In sum, as governing principles of corporeal nature, in the I60os the laws bridged mechanics and metaphysics; and as statements presumed to be universally true, they had help from a theory of a priori knowledge. An insidious problem will bring it all to an end.

\section{Limitations of early modern laws}

The current sense of 'mechanics' comes from Euler, who introduced it to rename what until then had gone by the 'science of motion'. Naturally and tacitly, they all took it to describe the motion of bodies in general-the ones that populate the world at all scales, from planets to microscopic particles. However, in advertising their theory of motion, early modern authors casually would leave out a crucial fact: bodies are extended, and yet the new science could not handle their motions qua extended objects. Specifically, none of the approaches born in the I6oos could describe quantitatively how every point of an extended body moves from one instant to the next, in response to agencies applied to the body.

Now why they could not handle it—what they lacked in that respect-is an important, difficult question; I give below a partial answer. Still, it is a fact that they were unable to handle the task. As illustration, consider two examples from the zenith of 17 th-century mechanics. Huygens in Horologium oscillatorium derived a key result that locates the "center of oscillation" in a compound pendulum. ${ }^{7}$ Then Newton in Principia (originally modeled after Huygens' approach) derived inter alia Kepler's Area Law that a body under a centripetal force around a fixed point sweeps out equal areas in equal times. But note how they both excise corporeal extension out of the account entirely. In Figure Ia, the circle $A B C$ represents an extended pendulum bob, attached to the $\operatorname{rigid} \operatorname{rod} F A$. $E$ is the gravity center, and $G$ is the bob's center of oscillation, whose distance from $A$

\footnotetext{
${ }^{7}$ A compound, or 'physical,' pendulum is one in which the bob is extended, and attached to a rigid massless rod swinging vertically from a suspension point. In contrast, a simple, or "mathematical," pendulum models the bob as an unextended mass point, attached to an inflexible massless string. The center-of-oscillation problem was to locate in a "physical" bob the point $H$ that swings isochronously with an equivalent mathematical pendulum. Specifically, let $P$ and $M$ be two equal-mass pendulums, compound and simple, respectively; and let $M$ oscillate with frequency $w$. The problem is to locate in $P$ the point $H$ that oscillates with frequency $w$.
} 
Huygens derives in his tract. Figure Ib presents Newton's derivation of the geometric quantity of the centripetal force on a planet in a Kepler orbit. $P$ stands for the mass center of an extended planet, and $S$ for the sun's center.
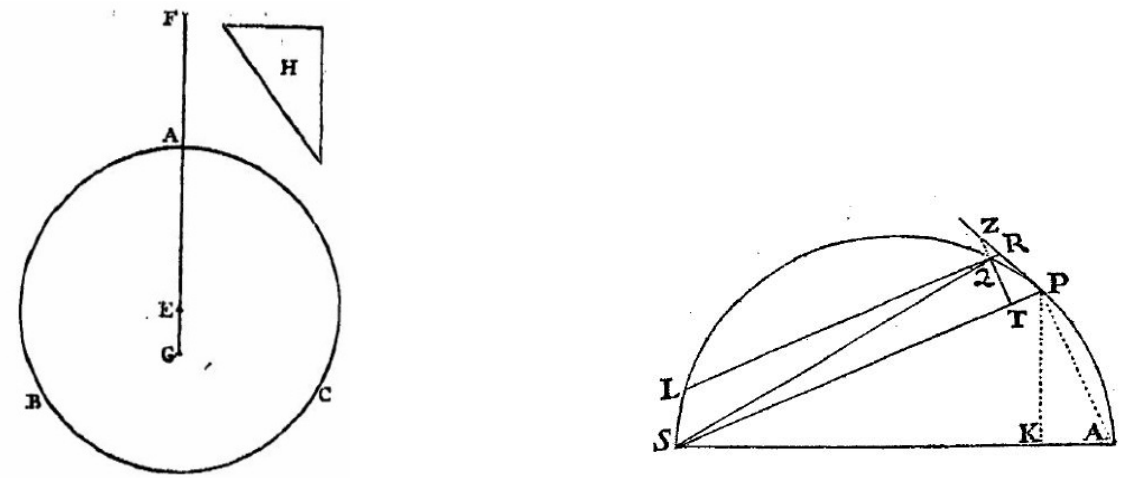

Figure I. Centroid mechanics. ${ }^{8}$

Though their respective topics (and targets of theory application) are bodies with shape and size, both Huygens and Newton quietly take the "representative point" approach. The pendulum bob above is an extended body, with matter distributed (perhaps unevenly) throughout its volume. Then each material point in the bob will move at a different linear speed from its neighbors, but Huygens does not know how to quantify the speed of every point in the bob. So he singles out just one point (the "center of oscillation"), whose motion he can derive from his theory. Likewise, the Area Law above was meant for planets and satellites, which-as extended, deformable bodies-engage also in complicated motions, like libration, precession, and tidal locking. But Newton does not know how to quantify the speed of every point in a planet as it performs those motions. So he singles out just one point (the center of gravity), whose motion he can derive from his theory.

Eventually the best theorists did grasp the laws' limitation. Euler gave it public expression in a paper (on the transmission of force in rigid bodies):

These principles are of no use in the study of motion, unless the bodies are infinitesimally small, hence the size of a point-or at least we can regard them as such without much error: which happens when the direction of the soliciting power passes through

\footnotetext{
${ }^{8}$ Left: from Huygens I673. Right: from Newton I 687.
} 
the center of gravity.... But if it does not pass through that center, we cannot determine the entire effect of these powers. That is all the more so if the moving body is not free, viz. it is constrained by some obstacle that depends on its structure. (Euler I745, §I7; my emphasis)

His friend, Daniel Bernoulli, then made the same point in $\mathbf{1 7 4 5}$, while emphasizing the insufficiency of Newton's original laws. Put in our terms, Euler's diagnosis was that, in extended bodies and constrained systems, the body's other parts and the constraints alter the motion of any point in the body, but there was no known general principle for quantifying all these kinematic effects.

\section{Mechanics: the great inflection}

The need for a general treatment-for any possible motion of any possible body - drove theorizing after I 740. Five decades later, it had caused a complete reformulation of mechanics. The locus of this transformation was continental Europe, with Paris and Berlin taking turns leading the way. The Europeans' quest for generality yielded three distinct dynamical laws; each law counted as basic, or fundamental, in the version of mechanics built on it. In this section, I survey them in turn.

\section{Laws of virtual work}

The most influential strand of new dynamical foundations began modestly in I 703 with Jakob Bernoulli's Démonstration générale du centre de balancement, a piece on the compound pendulum, aiming to infer from a safer foundation Huygens' formula giving the center of oscillation. ${ }^{9}$ Deep in that five-page paper, he buried the two seminal insights of modern analytic mechanics. The first regarded the statics of rigid weights; Bernoulli stated a generalized Lever Principle. Let $C, C, D$, and $D$ (in Figure 2) be masses attached to a bent lever free to oscillate about the fulcrum $A$. Resolve the impressed accelerations into radial and tangential components, respectively. Bernoulli's Lever Principle says the lever is in

\footnotetext{
${ }^{9}$ Huygens derived his formula from an energy principle; i.e., if the constraint (holding the parts of the pendulum bob together) were counterfactually dissolved, the free parts would re-ascend to the same height from which they fell (prior to the constraint being dissolved). In the I68os, Father Catelan, once Malebranche's private secretary, denied the truth of Huygens' premise. That led some (among them Jakob Bernoulli) to derive Huygens' formula from an incontestable dynamical principle. Cf. also Vilain 2000.
} 
equilibrium if the sum of each mass times its lever arm times the tangential component ( $C B$ or $E D$, respectively) add up to zero. ${ }^{\text {IO }}$ In our terms, the insight was that a system of constrained bodies is in equilibrium if the virtual work (compatible with the constraints) of the impressed forces is zero.

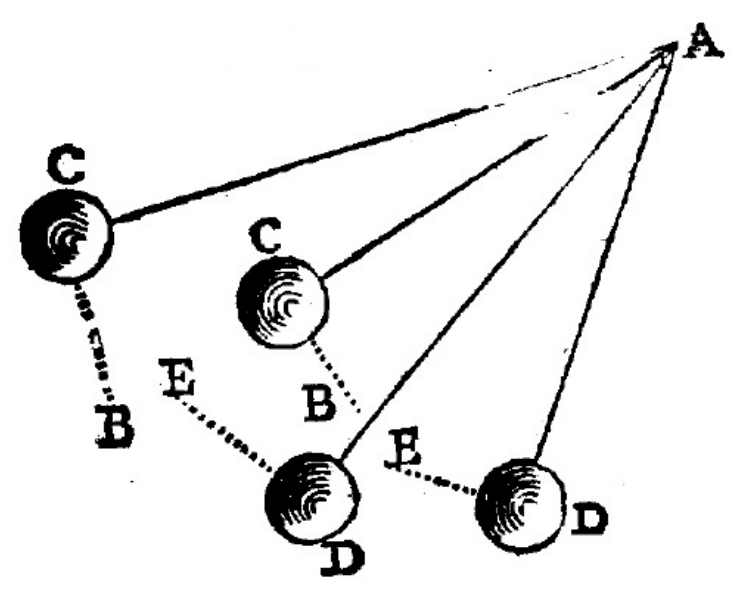

Figure 2. Bernoulli's Lever Principle. II

The second insight extends the virtual-work approach to a system in motion, not at mutual rest (which static equilibrium amounts to). Let some constrained masses $m_{1 \ldots i}$ move under the action of real forces $\mathbf{F}_{1 \ldots k}$. The forces and the constraints jointly cause each mass to undergo a net change in speed $\mathbf{a}_{i}$; that is, an effective acceleration. The general problem (and main difficulty of analytic mechanics) is to infer these accelerations. ${ }^{\text {I2 }}$ Bernoulli's breakthrough was heuristic; he found a way to turn a moving, dynamical system into a static one, which he knew how to treat (from his Lever Principle). He imagined that, in addition to the real forces, a set of fictive forces also act on the masses, equal and opposite

\footnotetext{
${ }^{\text {Io }}$ Note that the tangential components are signed magnitudes; e.g., (+) if they tend clockwise, and $(-)$ if counterclockwise.

${ }^{\text {II }}$ Appended to Bernoulli I703.

${ }^{\mathrm{I} 2}$ Here is why it is a problem. If the masses were kinematically free, Newton's Second Law would be sufficient: the resulting acceleration is in the direction of, and proportional to, the net force on every mass: $\mathbf{a}_{\text {real }}=\mathbf{f}_{\text {net }} / \mathrm{m}_{i}$. But the constraints prevent the actual-effective accelerations from being as above. Now constraint forces are generally unknown, so we cannot use the Second Law to compute their individual effects. That is why a different law is needed.
} 
to the products $m_{i} \cdot \mathbf{a}_{i}$. (For these fictive forces, we use the terms 'inertial' or 'kinetic reactions.') Bernoulli's second insight was that, in a moving system, the kinetic reactions balance the impressed forces-if they were real, they would cause equilibrium in the system of masses.

The two insights above became the two basic laws of Lagrange's mature theory. After a quiet, misattributed conversion to virtual-work methods in his youth, Lagrange re-derived all the equations of mechanics then known, from two principles that he stated as equalities. ${ }^{13}$ One is for statics, and it says that a system of bodies remains in equilibrium if the virtual work done by the system is zero. The other is for dynamics, and it asserts that, in a system of bodies, the net virtual work of the real-impressed forces and of the fictive, added-in-thought "kinetic reactions" vanishes. Lagrange does not spend much time stating his fundamental principle in words. Rather, he moves quickly to express it as a second-order, ordinary differential equation (Lagrange I788: I95). This formula allows Lagrange to derive the equations of motion for every point in a system of interacting bodies, whether free or subject to holonomic constraints.

For historiography, this version of mechanics holds a special interest. With d'Alembert's I743 treatise, the virtual-work approach became the canonical presentation of mechanics for the century or so in which France was the world's powerhouse in that discipline. ${ }^{\mathrm{I} 4}$ In contrast, the Newtonian approach (outlined next) was and remained the preferred version for a minority of one: Euler.

\section{Balance laws}

The same generic blind spot of 17 th-century theories (the motion of extended bodies under constraints) drove the rise of another formulation. It began with Euler's quest, throughout the I740s, to obtain equations of motion for a volume element $(d V)$ of an inviscid, incompressible fluid; for a point-mass bound to a rigid surface; and for a rigid body moving around a fixed point. By I758, Euler had come to think that one expression was enough to entail equations of motion for most mechanical systems regarded then as paradigmatic. Euler called his formula a "new principle of mechanics," and wrote it as:

\footnotetext{
${ }^{13}$ Fraser 1983 is an excellent overview of his first theory of mechanics, with an outline of d'Alembert's place in Lagrange's decision to move beyond it.

${ }^{14}$ Lagrange's principles of virtual work above remained the dynamical law for his greatly expanded second edition of Analytic Mechanics (I8I I-I3), for Laplace's five-volume treatise on celestial mechanics (I796-I 825), and for Poisson's bestseller, Physical Mechanics (I 833).
} 
That is, for every point in an extended body, the net force $\mathbf{f}$ equals the mass (at that point) times its resultant acceleration. Euler's principle functions as dynamical law in our sense: it entails equations of motion for several classes of extended bodies or systems. ${ }^{\text {I5 }}$

Subsequently, Euler discovered that his law (I) was not quite general. By itself, it was insufficient for deriving all the equations of motion needed. Specifically, for some systems (e.g., thin elastic rods, plates, and shells) the above law is not enough. Another principle must be added to it:

$$
\mathbf{t}=d^{2} \mathbf{1} / d t^{2}
$$

In words, the net torque on every point in a system of bodies equals the change in angular momentum by the system at every instant. Together, as he came to see, the equality formulas (I) and (2) are jointly sufficient for the generic task of deriving all the equations of motion.

After Euler, these two laws went dormant until the I830s, when Cauchy and Navier resurrected them for their mechanics of elastic bodies and viscous fluids. ${ }^{16}$ Nowadays we know them as the Balance Laws of Linear and Angular Momentum, respectively, because they relate the force and torque to the net change in those two kinematic quantities.

\section{Extremal laws}

One version of mechanics grew into maturity mostly unnoticed; its birth sparked much enmity and extravagant theologizing, which distracted many from grasping how this version rapidly grew into a serious contender for the title of general dynamics.

\footnotetext{
${ }^{15}$ To see some of the equations he obtained, cf. Stan (forthcoming) (for the rigid body); Darrigol and Frisch 2008 (for fluids without drag); and Verdun 20I 4 (for applications to celestial bodies). ${ }^{16}$ For that reason, some authors (e.g., Truesdell I99I; and his disciples) call them "Cauchy's Laws of Motion," to honor the French mathematician who showed them to be properly general.
} 
This approach arose in the mid-I740s, when Maupertuis found a way to derive two known results - the equilibrium condition for a straight lever and the kinematic equations for two-body impact-from a new law, which he called the Principle of Least Action. Around the same time, Euler used this principle for the harder task of deriving the equation of motion for a particle in Kepler motion around a fixed center of inverse-square attraction. Euler also devised a geometric version of the new calculus of variations needed to extend Maupertuis's new law. By $\mathrm{I} 753$, he also had discovered that in certain systems, the action is a maximum, not a minimum. ${ }^{17}$

Still, this was far from general, and it was anyone's guess just how much heuristic potential the least-action approach had. Though he was just a youth then toiling in obscurity at Turin, in 1760 Lagrange answered the question magisterially, in two steps. One step was a pithy version of the calculus of variations, restated in "analytic" (i.e., non-geometric) form. The other was to apply his calculus "to solving different problems in dynamics" (Lagrange I 762: 197). Specifically, he used it to state a "general principle," by reformulating the older Principle of Least Action as follows:

$$
\delta \int\left(m_{i} \mathbf{v}_{i}\right) d s=0
$$

From this variational principle, Lagrange then derived equations of motion for some eight different dynamical systems.

The upshot of these rapid developments is that, by 1790 , mechanics had witnessed a proliferation of basic laws, competing for supremacy over the principal task of theory; and that these new laws were all stated as differential equations, wholly unlike the I6oos laws of motion.

\section{Enabling factors: new mathematical structures}

Change in the aims of theory-building after 1740 cannot by itself explain the deep transformation that laws of nature undergo in the Enlightenment. There was another development, and I include it here as a co-explanans. It was the birth of four new notions in mathematics, specifically in the "new analysis," as

\footnotetext{
${ }^{17}$ For details on Maupertuis, Euler, and their context, see Pulte I989.
} 
they called it then. These notions jointly amounted to a result that helped change the logical structure of mechanics beyond anything its early fathers would have recognized. Here I survey these four concepts, and explain their relevance.

The first was the notion of a variable, then defined by appeal to an associated concept, that of a fixed quantity or constant value: "A variable quantity is a quantity that is indeterminate, or universal, and comprises every determinate value.... Thus a variable comprises in itself every number, be it positive or negative, integer or fraction, rational, irrational, or transcendent" (Euler I 748: 4). In practice, this notion behaved very much like our modern concept of a variable ranging over real numbers. ${ }^{18}$

The second discovery (an immense breakthrough in early modern theory generally) was the idea of a function. Several function-concepts emerged in the early Enlightenment, but the most important for my case was this one: "a function of a variable quantity is an analytic expression composed in any way whatsoever from that variable and numbers, or constant quantities" (Euler I748: 4). To explicate for the modern reader, an "analytic expression" is a combination of variables and constants, related by the operation-symbols of classical algebra $(+,-, \times, \div$, and $\sqrt{ })$. For instance, a function $\mathrm{f}$ of $x$ (i.e., $\mathrm{f} x$ ) might be expressed

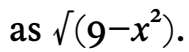

The third advance was a re-conceptualization of the essential notions of calculus, the derivative and the integral. Leibniz and his early disciples had conceived of both as magnitudes: $d x$ as an infinitely small increment in some variable quantity $x$; and the (definite) integral as a summatrix, or infinite-addition $\int$ of such increments. At mid-century, Euler reformulated these notions as two operators relating functions. In particular, for him the derivative was a function, $\mathrm{g}$, that stands to a given function $\mathrm{f}$ in the relation

$$
\mathrm{g}=d \mathrm{f}
$$

Conversely, Euler re-defined the integral as the anti-derivative; i.e., as the function $\mathrm{v}$ that stands to a given function, $\mathrm{w}$, in the relation

\footnotetext{
${ }^{18}$ Of course, scholars of this period did not have the notion of real numbers, or that it is a wellordered set. What they had were practical equivalents: the notions of integer, rational fraction of integers, magnitudes representable by Euclidean construction (i.e., irrationals), and also "transcendent" quantities, understood then as numbers that cannot be so constructed.
} 


$$
\mathbf{v}=\int \mathbf{w}
$$

A further and equally decisive innovation was Lagrange's extension of the calculus. He took an existing set of techniques (for solving so-called "isoperimetric" problems by geometric approaches) and turned it into the core of a general theory formulated algebraically (not geometrically, as variational problems and techniques had been stated, before him). Specifically, he created a new differential operator (the so-called variational $\delta$ ) that he defined purely algebraically, in terms of its commutation rules with $d$ and $\int$, which he likewise reconceived as operators on functions. ${ }^{19}$ For mechanics, Lagrange's innovation was an enormous step forward, since it allowed him to formulate the two laws [I] and [4] above, nowadays called the "variational principles" of classical mechanics. Both men were overt about the ways in which they had set the Leibnizian calculus on new foundations and extended it (cf. Euler I824: 4, Lagrange I788: I96).

The fourth innovation required the three above. Conceived as an "analytic expression" in the sense above, a function denotes a quantity, "so a function of a variable is itself a variable quantity" (Euler I748: 5). That is, a function just is a quantity - with the analytic expression making explicit the exact nature of its dependency on the underlying variable. ${ }^{20}$ This view of functions then enabled mathematicians to radically expand the concept of an equation. By I750, they had come to allow that, in an equation, the unknown variable can be a function-since a function always denotes a variable quantity. This new object is a "functional equation." Even more importantly, mathematicians allowed-by analogous reasoning-that differential operators on functions can appear in equations so reconceived:

To explain this matter, let $y$ be some function of $x$, and let it be defined by the quadratic equation $y^{2}+\mathrm{P} y+\mathrm{Q}=0 \ldots$ where $\mathrm{P}$ and $\mathrm{Q}$ are functions of $x \ldots$. In the same way as the finite equation $y^{2}+\mathrm{P} y+\mathrm{Q}=0$ displays a relation between $y$ and $x$, likewise a differential equation expresses a relation, or ratio, that $d y$ and $d x$ have to each other. (Euler I755: 24If.)

\footnotetext{
${ }^{19}$ Lagrange thought of $d$ and $\int$ as operators on functions. This was in line with his broader program of "analytic mathematics," i.e. of taking algebra (qua theory of operations on magnitudes) to be the general framework of all mathematics, with (analytic) geometry, arithmetic, and the calculus as species of this algebraic formalism. For lucid explanations, see Fraser I 990.

${ }^{20}$ That is the thought we convey by writing ' $\mathrm{f}(x)=y$ '. We regard $y$ as the dependent variable, with $x$ counting as independent relative to $y$. Then we specify their functional dependence by writing, e.g., that $\mathrm{f}(x)=x^{2}-5 \sqrt{x}$.
} 
That was the birth of differential equations as we think of them.

A qualification is needed: there were avatars of differential equations before I740. For instance, Newton's "fluxional" equations, which required a "fluent," or variable, to be inferred from its "fluxion," or derivative, qua a solution to the equation. But Newton's idea had a critical shortcoming: it was inextricably bound to a geometric configuration. (His fluent was the possible orbit of a pointparticle, and the fluxion was its instantaneous velocity.) In contrast, the Leibnizians' mathematical innovations above made it possible to study a differential equation as a self-standing object-unbound and independent from any geometric-kinematic representations it may have, such as orbits, worldlines, or families of curves traversable by matter points.

Here is how these innovations matter. Regarded from the vantage point of its mathematical structure, mechanics stops being a science of geometric representations (of motion patterns at specific scales); and it turns into a taxonomic system of differential equations, connected by genus-species relations. All versions of late-Enlightenment mechanics feature a generic differential expression, holding true of any mechanical system, irrespective of its geometry or mass/force distributions; and many specific expressions, one for each particular type of system. A paradigm example of the former is the Euler-Lagrange equation; and of the latter, the equation of motion for a binary star, a vibrating membrane, or a precessing gyroscope, and the equilibrium condition for a load-bearing truss, a capillary surface, or a buckling column.

It is easy for us moderns to miss the explanatory value of this particular development. It appears as a merely formal stage in the evolution of pure mathematics, with no obvious relevance to the science of nature. But that is a distorting effect of hindsight. The makers of mechanics did not see a clean-cut division of labor between pure and applied mathematics, as we do. Quite the opposite-many of their key results in now-pure mathematics were driven by problems and urgencies in mechanics.

\section{Academic foundations}

This far-reaching change in the status of the laws of motion occurred below the surface, as a deep undercurrent within cutting-edge theory construction carried out by leading mathematicians. My picture would not be complete, however, 
without a brief account of how some philosophers attentive to science saw matters then.

In general, philosophers missed the radical change happening at the time. The rapid increase in the formal complexity of mechanics, coupled with the philosophers' physical separation from the sites of theory construction, may well have contributed to the alienation of the two communities, philosophers and working mathematicians. ${ }^{2 \mathrm{I}}$ Perhaps as a result of this estrangement, the philosophers adhered to, and reinforced with novel doctrines, the 17 th-century notion of laws as metaphysical principles. Specifically, they restated the rationalists' two tenets above-i.e., that the basic laws of mechanics are corollaries of substance metaphysics, and that their truth rests on evidence from philosophy, not from empirical research.

Consider Christian Wolff, who was explicit that, in the science of motion, basic laws are the proper business of the philosopher:

The general principles of the rules of motion are called the laws of motion .... These days only a stranger to Mathematics is unaware that in the rules of motion there are general principles, from which these rules can be derived. These principles once established, the rules of motion, i.e. of impact, were proved from them in several ways. Mathematicians assume these laws without proof; but it behooves the Metaphysician to demonstrate them. (Wolff I73I: §§ 302-3; my emphasis)

Then he goes about his task. In Ontologia, he articulates-from mere concepts, with no empirical input-a vast theory of "simple" and "composite being." Then he applies it to bodies, in Cosmologia generalis, as follows. Bodies are composite, he argues, and eo ipso an ontological species of composite beings. Second, all bodies have-or at least mechanics takes them to have-three essential properties: extension, active force, and a passive force of inertia. Wolff theorizes from a strong commitment to the Principle of Sufficient Reason, and so here he sets out to uncover the sufficient reason for the three essential properties, which

\footnotetext{
${ }^{2 \mathrm{I}}$ Wolff and Kant worked in academia (at Halle and Königsberg, respectively) far from the Royal Academy of Berlin, the research institute where Maupertuis, Euler, and Lagrange worked. So they could not attend the presentation of mathematical work in progress, nor the ensuing discussions. And they lacked the skills to profit from the theorists' work; before the birth of the École Polytechnique in I792, training in advanced mathematics was available only through membership in informal networks of coaching and personal apprenticeship. (E.g., Jakob Bernoulli in Basel taught Jakob Hermann; then his brother, Johann, trained Euler and Maupertuis. D'Alembert and the young Lagrange were self-taught.) Émilie du Châtelet could well have come to learn and think about the foundations of Enlightenment mechanics, but she died young, unfortunately.
} 
he finds in the attributes of certain ontological ultimates that he calls synonymously "simple beings," "elements of bodies," or "physical monads." Bodies are aggregates of them. In turn, bodies qua aggregates are the subject-matter of two "laws of motion," namely a law of inertia and a law of action-reaction. Both are about two essential properties of body, active and passive force (of inertia). That is how ontology links up with mechanics in his doctrine: via his laws.

Kant is the last heir to this foundationalist picture of laws, his idiosyncratic jargon notwithstanding. This emerges in his 1786 tract, Metaphysical Foundations of Natural Science. The genus-concept of his ontology is "object of possible experience," which he divides into two species, according to the two perceptual channels ("inner" and "outer sense") whereby we access these objects. Any object of outer sense counts as matter, for Kant, and so it inherits all the topological, metric, and affine properties of space, insofar as it is the form of outer sense (which he thinks he has established in the Transcendental Aesthetic). Further non-empirical inferences-including an argument that motion is an attribute of all matter-result in a set of theses that he calls the "general doctrine of body," "metaphysics of material nature," and also "metaphysical foundation of natural science." Among these theses are three "mechanical laws": the conservation of mass, a law of inertia, and a principle of action-reaction.

First mechanical law: In all changes of corporeal nature, the total quantity of matter remains the same, without increasing or decreasing.... Second mechanical law: All change in matter has an external cause (every body continues in its state of rest or motion in a straight line at the same speed, unless it relinquishes this state, compelled by an external cause).... Third mechanical law: in all communication of motion, action and counter-action are always equal. (Kant I 9I I: 54I-5)

But Kant's confident talk above runs into the same problem as his two predecessors, Wolff and du Châtelet. Specifically, all three use the vocabulary of legislation-leges motus, lois du mouvement, and mechanische Gesetze-but they leave unclear what these laws are for; i.e., what they do within mechanical theory. More regrettably, Kant, the only philosopher to live through the great transformation in the laws' form and function described above, missed the chance to notice and reflect on it, an unfortunate victim of his deficient schooling.

And so a keen observer around I790 would have witnessed a paradoxical outcome: as mechanics became ever more able to handle real, extended bodies, it ceased to be a theory overtly about bodies. Kant had put forward his book at a "general doctrine of body," yet paid no attention to how it might connect with the most general, cutting-edge science of moving bodies-Lagrange's mechan- 
ics. The latter, in turn, had just published Mechanique analitique, a tract conspicuous through its absence of any explicit notion of body, even though it is a book about their motion, and their nature had long been a focus of philosophical inquiry in the two decades he had spent in Germany.

\section{Residual problems}

Beyond the unresolved paradox just mentioned, there are two more foundational worries induced by the silent metamorphosis of basic laws in the I 700 s. I outline them here, in the hope that future research will address them.

Causation. By relying on the same mathematical formalism, the basic laws of Enlightenment mechanics came to share an important trait. Specifically, the laws [I] through [3] above merely assert equalities between magnitudes, or that some quantity equals an algebraic combination of other quantities. That is all the more so as the laws emerged in response to the same need for equations of motion, despite their outward dissimilarity.

However, assertions of equality are neither material implications, nor if-then statements, nor any of the logical-syntactic vehicles used to predicate causeand-effect links or dependencies. And so the basic laws no longer count as causal principles after I790; nor do their local corollaries, the equations of motion. In effect, the laws no longer state facts about the basic causal powers of bodies, material substances, or their ontological analogues. If Enlightenment mechanics is still home to causal knowledge, it is not contained in the laws of that discipline.

This astonishing consequence-it really heralds the disappearance of causality from fundamental science-remained unnoticed until Russell came across it. Disappointingly for a modern reader, it eluded the I 8th century's great minds. Oblivious to the tectonic shift beneath their feet, philosophers and reflective men of science alike continued to refer to physics, whose rock bottom was then mechanics, as the "science of causes." But a hard-headed interlocutor might wonder where causes were hidden, seeing as they no longer reside in the laws.

Confirmation. The deep shift in the nature of laws had long-term epistemological consequences, too. Specifically, it is not clear what counts as evidence for basic laws any more. This grave problem-that laws lacked even prima facie warrant-had two causes.

First, the standard I7th-century sources of evidence for laws are no longer available to I8th-century mechanics. Top-down approaches to justification are 
now beside the point. As the laws no longer mention bodies or their causal actions, potential rationalist evidence (from substance metaphysics and philosophical theology) is irrelevant to the truth of the dynamical laws in the Enlightenment. ${ }^{22}$ Yet the bottom-up approach, which Newton's work epitomized, becomes out of reach. Here is why: Principia treated just a class of phenomenamotions at celestial scales-for which Newton's epistemic situation was twice privileged. First, he had vast amounts of data (going back to Babylonian astronomy) that informed him reliably on effective parameters and their key values; ${ }^{23}$ and second, the solar system (the main object of his theory) is remarkably wellbehaved compared to most species of mechanical system at terrestrial scales. ${ }^{24}$

Second, Enlightenment theorists were severely disadvantaged in both the respects that had favored Newton's search for evidence. For one, they had no reliable data with which to confront their equations of motion (so as to thereby extract evidence for the laws that entail the equations) ${ }^{25}$ For another, they were evidentially thwarted by the inexorable presence of large secondary effects, often due to agencies beyond the descriptive reach of the very theory in which the equation of motion belonged. ${ }^{26}$

In retrospect, we see that a key question-about the truth of the post-Newtonians' dynamical laws - soon became an enigma, avoided until late modernity.

\footnotetext{
${ }^{22}$ Caution: my points in this section pertain only to the law-statements $[\mathrm{I}]$ through $[3]$ above. As I explained, the philosophers worked with a different set of statements (which they kept calling laws). Their laws were amenable to top-down justification, and so of course much of their work in philosophy of physics aimed to obtain just this sort of a priori warrant.

${ }^{23}$ An effective parameter is some kinematic aspect that must show up in the equation of motion, and about which predictions in mechanics are made.

${ }^{24}$ For Newton's investigation, gravity is the only dominant source of motion; and when its secondary effects become relevant (e.g., in Saturn's attraction on Jupiter, in addition to the primary attraction on it by the Sun) Newton found a way to handle it by relying on perturbed-orbit methods, as in Book I, Section XI. Moreover, in gravitational setups, deformation rates are very slow, and so deformed configurations (e.g., the Earth's polar flattening) can be treated as quasistatic-hence Newton did not need an equation of motion for every part of the Earth (as an extended body) moving relative to the others under gravity and centrifugal force.

${ }^{25}$ The equations of $\mathrm{I} 8$ th-century mechanics describe infinitesimal deformations and instantaneous velocities at a point (inside an extended mechanical system). But measuring values for such local parameters requires very advanced technology_like high-resolution microscopy, fluorescent molecular doping, wind tunnels, and high frame-rate cameras-not available until lateVictorian physics and beyond. For instance, the earliest empirical data in elasticity theory (by Chladny, on the vibration of membranes) gets taken up by theorists after i 800 .

${ }^{26}$ E.g., elastic media available for experimentation deform irreversibly above a certain level of applied stress (i.e., they are really elastoplastic, not ideally elastic as the relevant theory-of elasticity-supposes them). Likewise, some liquid crystals respond to stresses differently when magnetic fields (which count as trans-mechanical agencies) are present.
} 


\section{Conclusions}

I have argued that our concept of laws of motion-namely, as dynamical lawsemerges in I8th-century mechanics. The chief driving factor for this shift was the need to extend mechanics beyond late-I6oos centroid theories. For this shift, the key enabling result was a development in mathematics, the rise of differential equations.

Thus an inflection point occurred circa $175^{\circ}$ as the science of motion exited its adolescence. Specifically, we see a deep shift in the form and status of laws of motion. The shift is where early modern mechanics turns into mechanics as we know it. Philosophically speaking, the inflection was genuinely deep: it changed the epistemic basis of mechanics; it altered its ontology in ways that we still struggle to grasp; and it had ripple effects on current ideas of what a law of nature is. In an important sense, the Long Scientific Revolution is still with us.

Acknowledgments. I am indebted to Katherine Brading for long, fruitful, and deeply illuminating conversations on the themes in my paper. For helpful suggestions and constructive criticism, I am grateful to David Miller, Zvi Biener, Brian Hepburn, Desmond Hogan, Adam Elga, Dan Garber, Gideon Rosen, Elliott Chen, Adam Chin, and Jeremy Heis. For stimulating discussions, I thank audiences at Iowa State, Princeton, UC Irvine, and Caltech.

\section{References}

Bernoulli, J. 1703. Démonstration générale du centre de balancement. Histoire de l'Academie Histoire de l'Académie royale des sciences, 78-84. Paris.

Brading, K. 20I I. On composite systems: Descartes, Newton, and the law-constitutive approach. Vanishing Matter and the Laws of Motion, eds. P. Anstey and D. Jalobeanu, I30-52. Routledge.

Calinger, R. 20I6. Leonhard Euler. Princeton University Press. 
Caparrini, S. \& Fraser, C. 2013. Mechanics in the I8th Century. The Oxford Handbook of the History of Physics, ed. J.Z. Buchwald and R. Fox, 358-405. Oxford University Press.

Darrigol, O. \& U. Frisch. 2008. From Newton's mechanics to Euler's equations. Physica D: Nonlinear Phenomena 237: I 855-I869.

Euler, L. I745. Dissertation sur la meilleure construction du cabestan. Pièces qui ont remporté le prix de l'Académie royale des sciences en 1741, 29-87. Paris.

Euler, L. I748. Introductio in analysin infinitorum, tomus I. Lausanne.

Euler, L. I755. Institutiones calculi differentialis, tomus I. St. Petersburg.

Euler, L. I824. Institutiones calculi integralis, volume I, ed. 3. St. Petersburg.

Ferraro, G. 2008. The integral as anti-differential. An aspect of Euler's attempt to transform the calculus into an algebraic calculus. Quaderns d'Història de l'Enginyeria IX: 25-57.

Fraser, C. I 983. Lagrange's Early Contributions to the Principles and Methods of Mechanics. Archive for History of Exact Sciences 28: 197-24I.

Fraser, C. 1990. Lagrange's analytical mathematics, its Cartesian origins and reception in Comte's positive philosophy. Studies in History and Philosophy of Science 21 : 24356.

Gallavotti, G. I983. The Elements of Mechanics. Springer.

Garber, D. I 992. Descartes' Metaphysical Physics. University of Chicago Press.

Hepburn, B. in print. The Quiet Scientific Revolution: problem solving and the I8th century origins of "Newtonian" mechanics. The Oxford Handbook of Newton, eds. Chr. Smeenk and E. Schliesser.

Jalobeanu, D. 20I I. The Cartesians of the Royal Society: the debate over collisions and the nature of body (I668-I670). Vanishing Matter and the Laws of Motion, eds. P. Anstey and D. Jalobeanu, I03-29. Routledge.

Jalobeanu, D. 2013. The nature of body. The Oxford Handbook of British Philosophy in the 17th Century, ed. Peter Anstey, 213-39. Oxford University Press.

Kant, I. I9II. Metaphysische Anfangsgründe der Naturwissenschaft. Gesammelte Schriften, Band IV, 480-557. Berlin: G. Reimer.

Lagrange, J.L. I762. Essay d'une nouvelle Methode pour determiner les maxima et les minima des formules integrales indéfinies. Application de la méthode précédente à la solution de différentes problèmes de dynamique. Mélanges de philosophie et de mathématique de la Société royale de Turin 2: 173-298. Turin.

Lagrange, J.L. I788. Méchanique analitique. Paris. 
Papastavridis, J. 2002. Analytical mechanics. Oxford University Press.

Pulte, H. I 989. Das Prinzip der kleinsten Wirkung und die Kraftkonzeptionen der rationalen Mechanik. Stuttgart: Steiner.

Stan, M. 2017. Newton's concepts of force among the Leibnizians. Reading Newton in early modern Europe, ed. M. Feingold \& E.A. Boran, 244-89. Brill.

Stan, M. in print. Euler, Newton, and foundations for mechanics. The Oxford Handbook of Newton, eds. Chr. Smeenk and E. Schliesser.

Truesdell, C. 1960. The rational mechanics of flexible or elastic bodies, I638-I788. Leonhardi Euleri Opera Omnia, Series II. Springer.

Verdun, A. 20I 4. Leonhard Eulers Arbeiten zur Himmelsmechanik. Springer. 